\title{
Frontières
}

\section{Remède, poison et charme magique}

\section{Johanne Collin et Joseph J. Lévy}

Volume 16, numéro 1, automne 2003

Remède ou poison?

URI : https://id.erudit.org/iderudit/1073754ar

DOI : https://doi.org/10.7202/1073754ar

Aller au sommaire du numéro

Éditeur(s)

Université du Québec à Montréal

ISSN

1180-3479 (imprimé)

1916-0976 (numérique)

Découvrir la revue

Citer ce document

Collin, J. \& Lévy, J. J. (2003). Remède, poison et charme magique. Frontières, 16(1), 5-8. https://doi.org/10.7202/1073754ar d'utilisation que vous pouvez consulter en ligne.

https://apropos.erudit.org/fr/usagers/politique-dutilisation/ 


\section{REMÈDE, POISON ET CHARME MAGIQUE}

\author{
Johanne Collin, Ph.D. \\ et Joseph J. Lévy, Ph.D., \\ conseillers sur le thème.
}

Le médicament est désigné en grec par le mot pharmakon auquel on attribuait jadis trois sens, ceux de remède, de poison et de charme magique. Il s'avère dès lors que cet objet pluriel revêt des significations non seulement diverses mais antinomiques; oscillant entre croyances populaires et magie d'un côté, rationalités scientifiques et savoirs professionnels de l'autre, mais également entre pouvoirs de vie et de mort. Il nous semblait dès lors pertinent d'aborder le thème dans une large perspective en l'examinant à partir des usages sociaux pluriels qu'il recouvre.

Les textes présentés dans ce numéro se modulent selon deux angles d'approche du phénomène médicament. Le premier envisage la substance chimique ou naturelle dans ses usages sociaux en tant qu'outil dont on se sert pour donner la mort ou maintenir la vie. Le caractère antinomique du médicament (poison et remède) est au cœur de cette approche. Il est envisagé comme outil pour donner la mort à travers des textes portant sur la place du poison dans l'histoire et la littérature (Anglade; Volant), mais également sous l'angle du médicament-poison dont le recours conduit au suicide ou à l'euthanasie (Pozo et al. ; Doucet). Susceptible de donner la mort, le médicament est également abordé comme outil thérapeutique qui guérit ou qui soigne. Son efficacité, et la perception qu'en ont les patients, ne va toutefois pas sans soulever la question des effets secondaires puissants qui souvent l'accompagnent (Argentier et al.; Proulx et al.).

Notre second angle d'approche consiste à appréhender le médicament comme vecteur de structuration des rapports sociaux. Il faut considérer, en effet, que le médicament est autant un objet social que technique. Il traverse en quelque sorte un cycle de vie au cours duquel son rôle et ses finalités se construisent socialement et culturellement en même temps qu'il transforme les dynamiques et rapports sociaux dans lesquels il s'inscrit (Van der Geest et al., 1996; Nichter et Vuckovic, 1994). Signe tangible du pouvoir de guérir des médecins et incarnation de la technologie moderne, le médicament représente beaucoup plus qu'un outil thérapeutique. II constitue l'un des vecteurs de structuration de nos rapports à la maladie (Mongeau et al.) et à la vieillesse (Collin). Il médiatise et transforme la relation entre consommateur et producteur, patient et professionnel mais éventuellement aussi entre individu et société (Otero, Saint-Germain). On peut également envisager ses effets structurants sur les pratiques professionnelles (Lafortune $e t$ al.) ainsi que sur les pratiques de soins et systèmes de santé, notamment à travers l'usage politique des tensions que recouvre l'opposition entre biomédecine et thérapeutiques traditionnelles (Monnais ; Laplante). Il importe dès lors de se pencher plus attentivement sur chacun de ces angles d'approche.

\section{LES MÉDICAMENTS ET LES POISONS : SOURCES DE MORT ET DE VIE}

Les poisons ont joué un rôle significatif dans l'histoire de l'humanité et ce, depuis les temps les plus reculés. L'histoire est ainsi parsemée d'intrigues où le poison, instrument du pouvoir politique, contribue à l'élimination de rivaux ou de témoins gênants ou est utilisé comme moyen d'éviter l'esclavage, l'exil ou la torture. La mort de Socrate, à la suite de l'ingestion d'une coupe de ciguë, ou celle de Cléopâtre attribuée à la morsure d'un aspic ou d'un poison que, selon Plutarque, elle avait caché dans une épingle à cheveux creuse, sont autant d'épisodes célèbres. Les accusations d'empoisonnement, réelles ou imaginaires, parsèment également les chroniques du Moyen Âge et des périodes ultérieures comme en témoignent les personnages de Lucrèce Borgia, empoisonneuse, mise en scène par Victor Hugo, ou la marquise de Brinvilliers. 
Mais l'usage du poison dans l'histoire ne s'est certes pas limité à servir les desseins individuels. On le retrouve également employé comme moyen de résistance et de rébellion de la part des esclaves africains, où il est dans les colonies antillaises qui l'utilisaient pour assassiner leurs maîtres. Lors des guerres mondiales, les armes chimiques se fondaient sur l'utilisation de poisons, comme le fameux gaz moutarde ou ypérite pendant la Première Guerre mondiale ou le zyklon B lors du génocide des Juifs et des Tsiganes. Malgré les règlements internationaux interdisant l'utilisation des poisons ou des armes empoisonnées, ceux-ci ont continué de servir d'instruments de combat dans des conflits contemporains ou dans les attaques terroristes, ce qui fut le cas en 1995 lors de l'attaque au gaz sarin dans le métro de Tokyo par des membres d'une secte religieuse dévoyée.

Au-delà de la réalité historique, les poisons ont frappé l'imagination populaire et inspiré les œuvres littéraires. Est-il aussi nécessaire de rappeler le rôle central des poisons (arsenic, strychnine, cyanure, les oxydes de carbone, les alcaloïdes, etc.) ou des médicaments (barbituriques) dans les fictions littéraires, en particulier les romans policiers ou d'espionnage. On peut ici penser à des œuvres d'auteurs comme Agatha Christie, Conan Doyle, Georges Simenon ou Ian Fleming.

Deux des textes présentés ici illustrent, chacun à leur façon, les usages des poisons à travers la littérature et l'histoire. Georges Anglade, écrivain d'origine haïtienne, présente ainsi l'une de ses Lodyans, une forme littéraire originale et propre à Haïti qui se caractérise par une concision recherchée, et qui met en scène un cas d'empoisonnement. Il situe, par la suite, le rôle du poison dans la genèse de la société haïtienne, avec la figure historique de Makandal, l'un des meneurs de la lutte pour les revendications nationales haïtiennes et pour qui le poison constituait une arme politique privilégiée dans la lutte contre les planteurs français au pouvoir. Ce personnage, tout comme le thème du poison en lui-même, occupe une place significative dans plusieurs œuvres romanesques de la littérature haiitienne dont Georges Anglade esquisse la généalogie.

Le thème du poison, tel qu'il est inscrit dans l'histoire de la mort de Socrate provoquée par l'ingestion d'une coupe de ciguë, sert de point de départ à la réflexion philosophique d'Éric Volant. Celui-ci situe cet épisode dans le courant des préoccupations philosophiques de la Grèce antique et des réflexions de Nietzsche sur cet acte pour en faire ressortir les enjeux. Il soulève en effet tout un ensemble de questions liées aux rapports entre l'âme et le corps et à la valeur

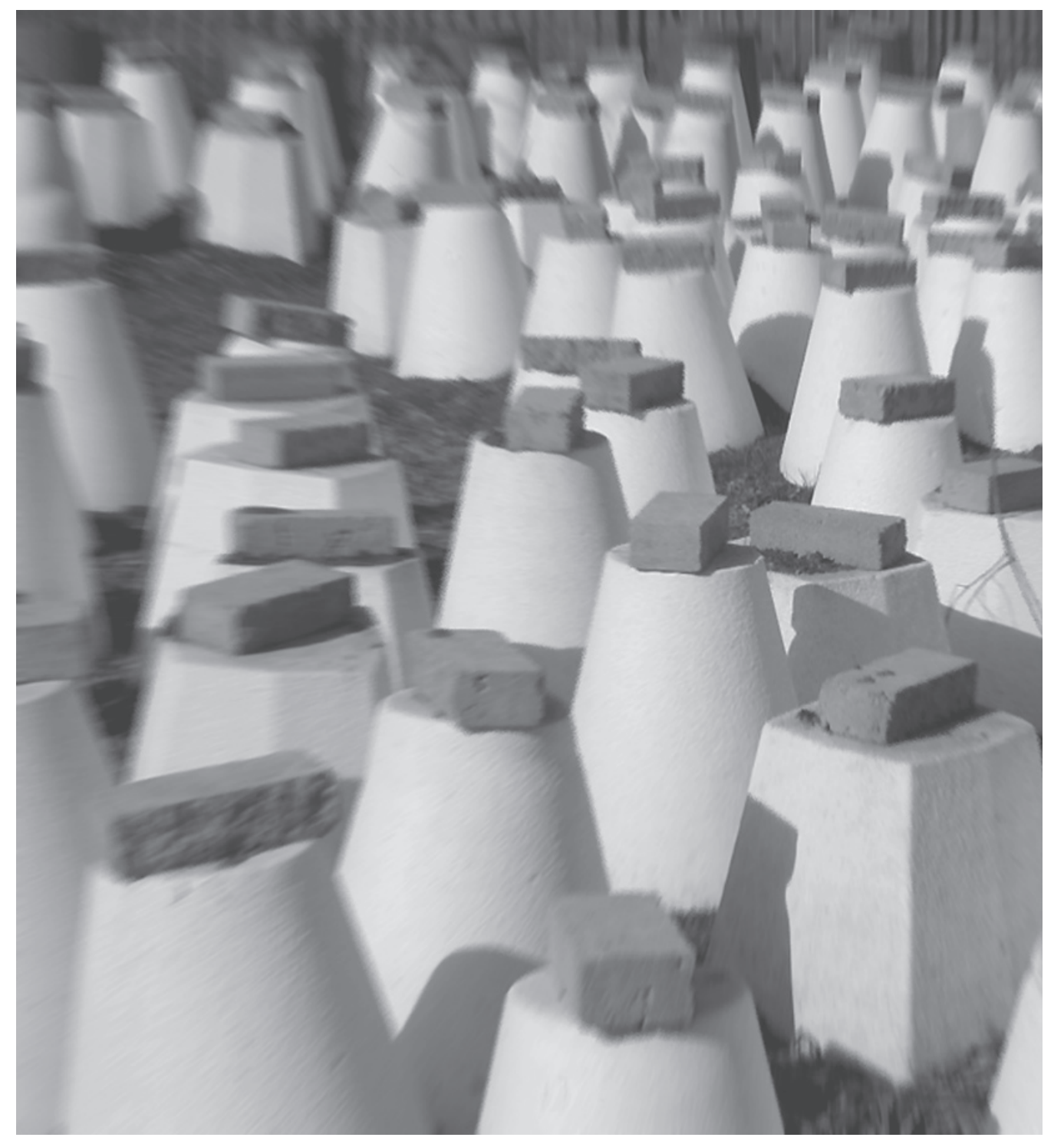

accordée à ce dernier. Mépris du corps ? Geste politique démontrant sa liberté ? Foi dans l'immortalité ? Refus de l'exil? Acte de raison en continuité avec sa philosophie? Le geste mortel de Socrate est mis en contraste avec la modalité d'affrontement de la mort chez Nietzsche qui s'accompagne d'un lent délabrement physique et mental.

Deux textes abordent, quant à eux, la problématique des médicaments-poisons et leurs effets dans les processus thanatiques. Michel Pozo, Brian Mishara et Anne SecondPozo analysent ainsi la place des médicaments dans le suicide et montrent que leur usage est de plus en plus important. Cette tendance est cependant modulée par de nombreux facteurs (âge, genre, disponiblité et facilité d'accès, etc.). Parmi les médicaments utilisés, les psychotropes et les analgésiques / antalgiques sont les plus nombreux. Les représentations et les croyances entourant les effets des médicaments dans le processus thanatique peuvent aussi intervenir en valorisant une mort douce et facile. Cette analyse suggère la complexité des rapports entre les médicaments et les conduites suicidaires, signalant une fois de plus les dimensions contradictoires des médicaments, source de guérison ou de mort.

Hubert Doucet, quant à lui, se focalise sur les enjeux éthiques entourant la question de l'euthanasie en montrant la diversité des points de vue en fonction des sensibilités nationales. Bien qu'il n'aborde pas directement la question du médicament, celle-ci peut s'inscrire en filigrane des débats que soulève l'euthanasie. Doucet souligne les questionnements quant aux objectifs de la profession médicale. S'agitil de maintenir la vie à tout prix ou de reconnaître la place de la mort et du mourir, les valeurs rattachées à la qualité de la vie et à la dignité et donc à la mort volontaire ? Les arguments complexes qui accompagnent la reconnaissance ou non de l'euthanasie sont aussi présentés, montrant les limites des discours contemporains sur cette question cruciale. 


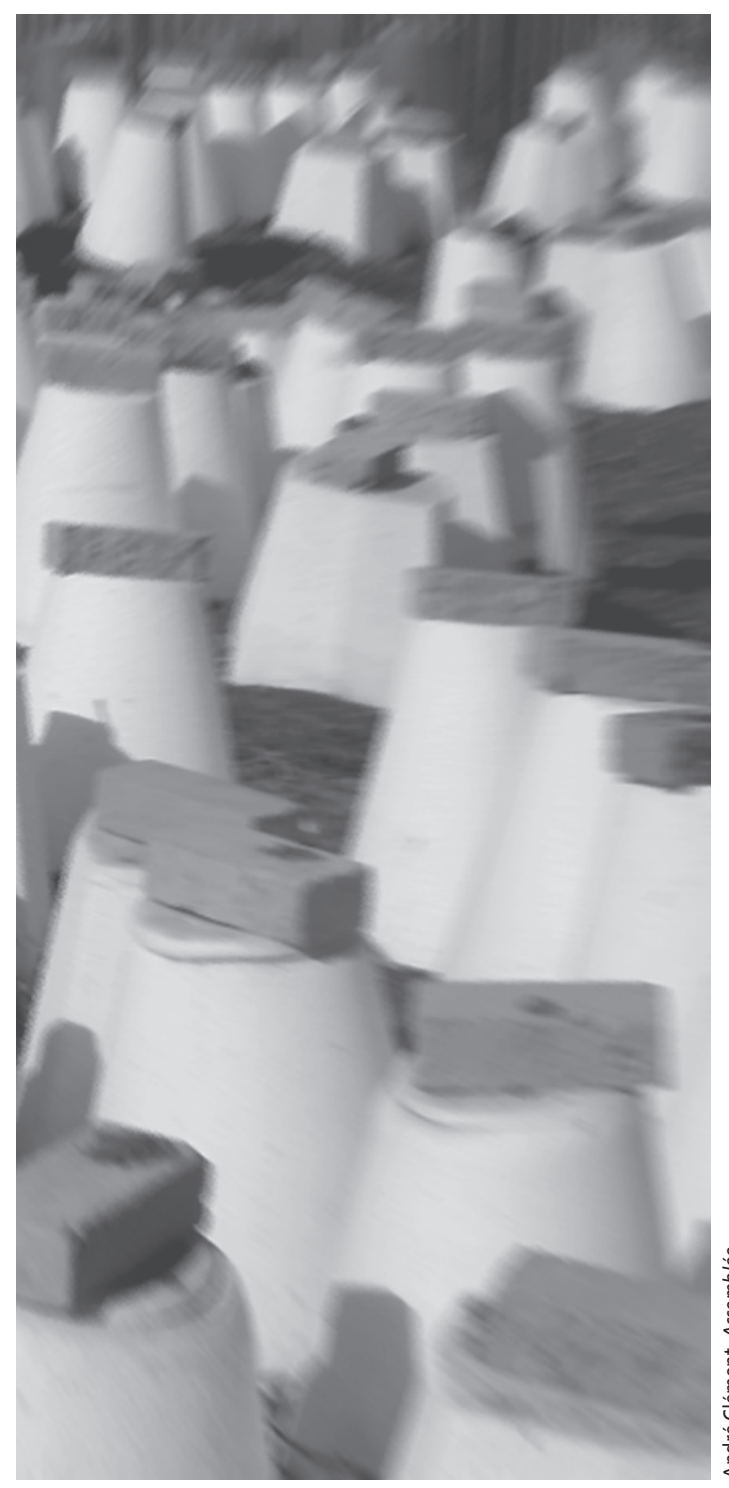

En contrepartie, les médicaments sont aussi source de vie. Prenant le relais de l'arsenal thérapeutique complexe mais archaïque et peu efficace qui caractérise la fin du XIX ${ }^{\mathrm{e}}$ siècle, les médicaments modernes constituent en quelque sorte une révolution thérapeutique dans le traitement des maladies physiques et mentales. Depuis la découverte des sulfamides, en passant par celle de l'insuline, des premiers antibiotiques et des neuroleptiques, les thérapies se sont diversifiées pour s'attaquer aux pathologies modernes (ou de la modernité) : troubles mentaux, maladies cardiovasculaires, cancers, dysfonctions sexuelles et $\mathrm{VIH} /$ sida. Les progrès majeurs introduits par les médicaments modernes s'accompagnent cependant d'effets pervers liés à ces consommations : qu'il s'agisse des effets secondaires des médicaments eux-mêmes ou de l'accroissement des risques d'interactions médicamenteuses et de surconsommation des médicaments.

Cette dualité remède-poison est dès lors réintroduite, même lorsqu'il est question du versant positif de ces produits. De fait, les effets thérapeutiques qui caractérisent le médicament par rapport à d'autres substances sont affaire de dosage. Les doses thérapeutiques et les doses toxiques d'un médicament s'inscrivent dans un continuum ; ce sont les variations en concentration ou en quantité qui déterminent dans quelle mesure les effets «bénéfiques »c'est-à-dire recherchés au niveau thérapeutique - vont l'emporter sur les effets négatifs - c'est-à-dire non recherchés et que l'on désigne généralement comme les effets secondaires. Comme le faisait justement remarquer Montagne (1997, p. 152) : «Il semble exister une croyance répandue qu'une drogue produit un seul effet, ou un effet primaire considéré comme le principal avec une connotation positive. Si d'autres effets sont ressentis, ils sont tenus pour secondaires, avec cette fois une connotation négative. En fait, toute drogue produira un ensemble de changements cellulaires, physiologiques et psychologiques chez qui en fait usage. »

Abordant cette dualité du médicament, l'article de Stéphanie Argentier et al. se penche ainsi sur les représentations des nouvelles thérapies contre le VIH / sida qui ont été accueillies par les milieux biomédicaux comme une avancée majeure dans la lutte contre l'épidémie, en contribuant à : réduire la mortalité et en accroissant l'espéहै rance de vie. Les expériences des patients montréalais, hommes ou femmes, mettent 䓂 en relief l'apparition d'effets secondaires majeurs chez plusieurs personnes vivant avec le VIH, ce qui contribue à remettre en question l'efficacité de la thérapie, alors que les représentations de ces médicaments oscillent entre des perspectives contradictoires: source de vie et d'espoir pour certains, ils sont pour d'autres associés, au contraire, à la maladie et à la mort qu'ils devaient contribuer à effacer. Cette ambivalence montre bien les écarts entre les discours biomédicaux et expérientiels et met en évidence la perception polysémique de ces médicaments modernes.

L'article de Michelle Proulx et al. sur la construction du risque chez des hypertendus qui ne suivent pas avec fidélité leur traitement montre que, parmi ces patients, les risques associés à l'hypertension artérielle sont considérés comme faibles sinon nuls et ce, en raison de l'absence de symptômes susceptibles de signaler au patient l'importance de suivre le protocole thérapeutique. La non-adhésion au traitement est aussi liée aux représentations problématiques du médicament considéré comme une source d'effets néfastes sur l'organisme et perçu comme un produit toxique. À ces représentations viennent s'ajouter les contraintes du régime thérapeutique qui contribue à dissuader les patients de le suivre avec fidélité. Cette analyse met aussi en relief l'ambivalence face aux médicaments qui recoupe les données d'autres études.

\section{LES MÉDICAMENTS COMME VECTEURS DE STRUCTURATION DES RAPPORTS SOCIAUX}

La symbolique du médicament transcende les propriétés thérapeutiques de ces substances. Pellegrino (1976) note que peu d'expériences humaines comportent une charge symbolique aussi forte que le geste ordinaire d'ingérer des médicaments. Les représentations sociales du médicament constituent à la fois des interprétations de la réalité concrète et des phénomènes complexes qui ont un sens pour les acteurs. Par conséquent, elles orientent leurs pratiques et façonnent les politiques et institutions qui les sous-tendent. Ces représentations s'inscrivent alors dans un double travail de traduction et de construction de la réalité : travail de traduction à travers le regard direct qu'elles procurent sur les perceptions, attitudes et valeurs d'acteurs inscrits dans des rapports sociaux spécifiques face aux médicaments et donc sur les processus symboliques organisant ces rapports ; travail de construction à travers les pratiques sociales qu'elles contribuent à forger. Représentations et effets tangibles associés aux médicaments concourent donc à moduler et structurer les rapports sociaux entre groupes et entre individualités et collectivités, comme en témoigne la deuxième série de textes que nous présentons ci-dessous.

Suzanne Mongeau et al. se penchent sur les patients cancéreux face à leur traitement. Ils mettent ainsi en évidence des relations problématiques avec le personnel soignant et un certain évitement dans l'expression de la souffrance. Les traitements, fortement investis, malgré les effets secondaires, s'inscrivent dans une perspective associée à un combat contre la maladie et constituent un mode de structuration du rapport à la maladie. Leur arrêt en cas de progression de la maladie est considéré comme une défaite et entraîne un sentiment d'impuissance et d'abandon face à une mort que l'implication dans les soins palliatifs, et donc le passage à un autre type de médication, vient confirmer.

Johanne Collin met en évidence, quant à elle, le rôle structurant des médicaments dans notre rapport au vieillissement et à la mort. Nos sociétés modernes avancées se caractérisent notamment par un rapport au temps ancré dans le présent et un rapport au corps visant l'ultime de l'apparence et $\mathrm{du}$ confort; caractéristiques qui ne sont guère propices à la valorisation de ce qu'incarne la vieillesse. Dans cette phase de l'existence où le temps - à travers son travail 
sur le corps - devient une donnée incontournable, le médicament occupe une place centrale. Il représente le rempart contre la mort, l'assurance d'un contrôle, d'une certaine prise sur l'évolution de la maladie chronique. Son recours devient alors d'autant plus incontournable pour les personnes âgées, qu'il ne fait que traduire, de façon exacerbée, la préoccupation majeure que nourrit l'individu en contexte de modernité avancée, pour sa corporéité, son apparence, son confort et sa santé.

La réflexion sur le lien social, déjà esquissée à travers le rapport entre médicament et vieillesse, se poursuit à partir de deux textes portant sur psychotropes et société. L'individualité de masse qui caractérise la modernité avancée conduit à se pencher sur l'exigence d'autonomie et de performance qu'impose la normativité contemporaine; normativité qui soulève la question de la mort du sujet, à travers la standardisation des comportements et des émotions. Le psychotrope contribue ici largement à structurer les rapports entre individu et société.

L'article de Marcelo Otero s'attarde à la question des antidépresseurs pour en situer l'usage dans le contexte des transformations socioéconomiques que connaissent les sociétés contemporaines. L'instabilité qui caractérise ces sociétés contribuerait à amplifier les problèmes d'adaptation des populations qui ont alors recours à une gestion thérapeutique qui vise non pas un sujet qui exprime ses malaises mais un corps dont il s'agit de moduler l'humeur et les capacités à agir soit par une intervention psychothérapeutique, soit par le recours à des médicaments psychotropes, en particuliers les antidépresseurs, une tendance associée à la reformulation des troubles et des diagnostics psychiatriques.

Christian Saint-Germain, dans son article sur les antidépresseurs, montre aussi comment ces médicaments jouent un rôle central dans le déploiement du contrôle biomédical contemporain qui, s'inscrivant dans un imaginaire narratif et publicitaire et se fondant sur des processus de marketing, vise à la standardisation du comportement et la normalisation de la pensée dans une tentative problématique de favoriser le bonheur, une valeur inscrite dans l'idéologie dominante. Cette analyse critique met en évidence comment les médicaments antidépresseurs participent d'un imaginaire social puissant dont les finalités ne font plus référence à une subjectivité assumée mais à une standardisation mentale et comportementale.

Le médicament comme vecteur de structuration des rapports professionnels et éventuellement de redéfinition des frontières et des juridictions de pratiques est un thème abordé par Denis Lafortune et al. Les auteurs s'interrogent sur la question des risques suicidaires chez les jeunes en centres jeunesse et les enjeux pratiques et éthiques liés à l'usage des substances pharmacologiques. Les intervenants sont d'accord avec la mise en place d'un protocole d'intervention qui stipule qu'un médecin doit être impliqué dès qu'un jeune présente le moindre risque suicidaire, mais plusieurs s'interrogent sur les conséquences du recours aux médicaments psychotropes qui accompagne cette prise en charge, sur leur rôle professionnel et leur responsabilité dans le suivi de l'enfant. Le manque d'informations sur les effets des médicaments; les possibilités de surdoses et d'interactions avec d'autres substances, le suivi médical et les effets démobilisants sur l'équipe et l'entourage sont autant de questions que soulève le recours aux médicaments.

Dans son article sur les fonctions de l'arsenal thérapeutique dans le Viêtnam colonisé, Laurence Monnais montre le rôle structurant de celui-ci par rapport aux pratiques de soins et à l'établissement d'un système de santé en régime colonial. Elle analyse comment un événement associé à l'empoisonnement de soldats français par leurs subalternes vietnamiens conduit à la mise en place d'un contrôle de la pratique médicale et pharmaceutique locale par l'administration coloniale. L'incident donne lieu au déploiement d'un discours sur les dangers des pharmacopées locales. Leur dimension toxique, alliée à l'automédication, est ainsi opposée à la médecine occidentale scientifique, efficace et fondée sur une connaissance précise des substances.
De ce fait, ce contrôle devient l'expression d'une mission civilisatrice associée à l'interdiction de l'usage de certains produits pharmaceutiques et de pratiques médicales jugées non conformes aux canons occidentaux, contribuant ainsi à restructurer les pratiques de santé.

L'étude de Julie Laplante sur la dynamique de l'usage des médicaments et des remèdes locaux en Amazonie analyse la contribution de ces deux classes aux enjeux associés à la vie et à la mort et met en relief comment chacun des paradigmes (biomédical ou traditionnel) structure le rapport à la mort, envisagée comme conséquence de l'inaccessibilité, soit aux médicaments, soit aux pouvoirs des chamans maîtrisant les propriétés des plantes. À partir d'exemples, elle met en évidence les frontières floues qui séparent les propriétés curatives et mortifères des produits utilisés, et ce dans un contexte de changements rapides du champ des représentations de la maladie liés à la pénétration des modèles biomédicaux occidentaux remettant en question les savoirs locaux.

Ces textes, dans leur ensemble, mettent en relief la complexité des rapports aux poisons, aux remèdes et aux médicaments dont les représentations et les usages soulèvent des questions liées à la dialectique mort-vie.

\section{Bibliographie}

MONTAGNE, M. (1997). «De l'activité pharmacologique à l'usage des drogues: la construction des connaissances sur les psychotropes». Santé mentale au Québec, 22 (1), 149-163.

NICHTER, M. et VUCKOVIC, N. (1994). "Agenda for an anthropology of pharmaceutical practice». Social Science and Medicine 39(11), 1509-1525.

PELLEGRINO, E. D. (1976). «Prescribing and Drug Ingestion: Symbols and Substances». Drug Intelligence \& Clinical Pharmacy. 10, 624-30.

VAN DER GEEST, S., HARDON, A. et WHYTE, S.R.(1996). "The anthropology of pharmaceuticals : A biographical approach ». Annual Review of Anthropology, 25, 153-78. 